\title{
Acidovorax temperans
}

National Cancer Institute

\section{Source}

National Cancer Institute. Acidovorax temperans. NCI Thesaurus. Code C86088.

A species of aerobic, Gram negative, rod shaped bacteria assigned to the phylum

Proteobacteria. This bacteria is motile by a single polar flag ellum, oxidase positive, chemoorganotrophic, resistant to penicillin and can reduce nitrites. A. temperans is commonly found in soil. 